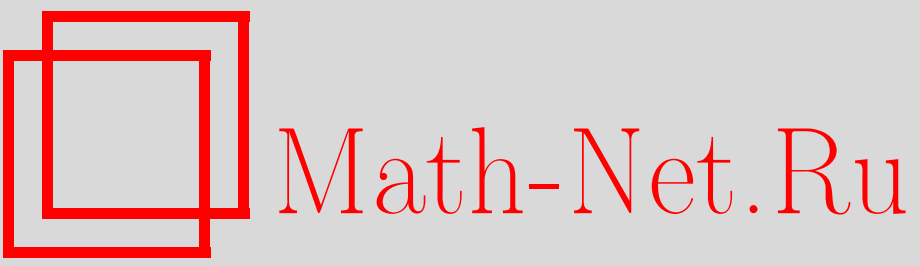

Я. А. Сатин, А. И. Зейфман, А. В. Коротышева, О скорости сходимости и усечениях для одного класса марковских систем обслуживания, Теория вероятн. и ее примен., 2012, том 57, выпуск 3, 611-621

DOI: https://doi.org/10.4213/tvp4469

Использование Общероссийского математического портала Math-Net.Ru подразумевает, что вы прочитали и согласны с пользовательским соглашением

http://www. mathnet.ru/rus/agreement

Параметры загрузки :

IP : 54.224 .135 .184

26 апреля 2023 г., 13:32:24 
6. Chan H., Kutoyants Y. On threshold estimation for some TAR models. Preprint, 2010.

7. Dachian S., Negri I. On compound Poisson processes arising in change-point type statistical models as limiting likelihood ratios. - Statist. Inference Stoch. Process., 2011 , v. 14 , № 3 , p. $255-271$.

8. Dufresne D. The distribution of a perpetuity, with applications to risk theory and pension funding. - Scand. Actuar. J., 1990, № 1-2, p. 39-79.

9. Fujii T. An extension of cusp estimation problem in ergodic diffusion processes. Statist. Probab. Lett., 2010, v. 80, № 9-10, p. 779-783.

10. Gjessing H., Paulsen J. Present value distributions with applications to ruin theory and stochastic equations. - Stochastic Process. Appl., 1997, v. 71, № 1, p. 123-144.

11. Голубев Г. К. О вычислении эффективности оценки максимального правдоподобия при наблюдении разрывного сигнала в белом шуме. - Проблемы передачи информации, 1979 , т. 15, № 3, с. 61-69.

12. Gushchin A.A., Küchler U. On estimation of delay location. - Statist. Inference Stoch. Process., 2011, v. 14, № 3, p. 273-305.

13. Ибрагимов И. А., Хасьминский Р. З. Асимптотическое поведение обобщенных байесовских оценок. - Докл. АН СССР, 1970, т. 194, с. 257-260.

14. Ибрагимов И. А., Хасьминский Р. З. Оценка параметра разрывного сигнала в белом гауссовском шуме. - Проблемы передачи информации, 1975, т. 11, № 3, с. 3143.

15. Ibragimov I. A., Has'minskii R.Z. Statistical Estimation. Asymptotic theory. New York-Berlin: Springer-Verlag, 1981, 403 p.

16. Kutoyants $Y$. Statistical Inference for Ergodic Diffusion Processes. London: SpringerVerlag, 2004, $481 \mathrm{p}$.

17. Pitman E. J. G. The estimation of the location and scale parameters of a continuous population of any given form. - Biometrika, 1939, v. 30, p. 391-421.

18. Port S.C., Stone C.J. Fisher information and the Pitman estimator of a location parameter. - Ann. Statist., 1974, v. 2, № 2, p. 225-247.

19. Rubin H., Song K.-S. Exact computation of the asymptotic efficiency of maximum likelihood estimators of a discontinuous signal in a Gaussian white noise. - Ann. Statist., 1995, v. 23, № 3, p. 732-739.

20. Stein C. The admissibility of Pitman's estimator of a single location parameter. Ann. Math. Statist., 1959, v. 30, p. 970-979.

21. Yor M. Exponential Functionals of Brownian Motion and Related Processes. Berlin: Springer-Verlag, 2001, 205 p.

Поступила в редакцию

1.IX.2011

(C) $2012 \Gamma$

САТИН Я. А.*, ЗЕЙФМАН А. И.**, КОРОТЫШЕВА А.В.*

\section{О СКОРОСТИ СХОДИМОСТИ И УСЕЧЕНИЯХ ДЛЯ ОДНОГО КЛАССА МАРКОВСКИХ СИСТЕМ ОБСЛУЖИВАНИЯ ${ }^{1)}$}

В статье рассматриваются нестационарные марковские цепи с непрерывным временем, описывающие марковские системы массового обслуживания с групповым поступлением и обслуживанием требований.

* Вологодский государственный педагогический университет; e-mail: yacovi@ mail.ru; a_korotysheva@mail.ru

** Вологодский государственный педагогический университет, Институт проблем информатики РАН, Институт социально-экономического развития территорий РАН; a_zeifman@mail.ru

1) Работа выполнена при поддержке РФФИ (гранты 11-01-12026-офи-м, 12-0700115-a, 12-07-00109-a). 
В предположении, что интенсивности поступления и обслуживания требований не зависят от текущего состояния системы, указан метод получения точных по порядку оценок скорости сходимости к предельному режиму и исследованы вопросы, связанные с приближенным построением предельных характеристик системы.

Ключевые слова и фразы: марковские системы обслуживания, нестационарные марковские цепи с непрерывным временем, скорость сходимости, точные оценки, усечения.

1. Введение. Классы систем массового обслуживания, описываемых процессами рождения и гибели (стационарными и нестационарными, с катастрофами), изучались начиная с 70-х годов двадцатого века многими авторами (см., например, [1]-[5], [7], [10], [12], [18], [19], [21]). С помощью методов, разработанных, в основном, одним из авторов настоящей статьи (подробное изложение приведено в [6], [9], [11], [15], [17]), для таких систем удалось получить точные оценки скорости сходимости и устойчивости.

Оказывается, этот же подход можно применить и к существенно более общему классу систем обслуживания.

Рассмотрим систему массового обслуживания, число требований в которой описывается нестационарной марковской цепью с непрерывным временем и счетным пространством состояний $E=\{0,1, \ldots\}$, причем требования могут поступать и обслуживаться группами.

Пусть $X=X(t), t \geqslant 0$, есть число требований в системе обслуживания $(0 \leqslant$ $X(t)<\infty)$.

Обозначим $p_{i j}(s, t)=\operatorname{Pr}\{X(t)=j \mid X(s)=i\}, i, j \geqslant 0,0 \leqslant s \leqslant t$, переходные вероятности процесса $X=X(t)$, а $p_{i}(t)=\operatorname{Pr}\{X(t)=i\}$ - его вероятности состояний.

Будем всюду далее предполагать, что

$$
\operatorname{Pr}(X(t+h)=j \mid X(t)=i)= \begin{cases}q_{i j}(t) h+\alpha_{i j}(t, h) & \text { при } j \neq i, \\ 1-\sum_{k \neq i} q_{i k}(t) h+\alpha_{i}(t, h) & \text { при } j=i,\end{cases}
$$

причем все $\alpha_{i}(t, h)$ суть $o(h)$ равномерно по $i$, т.е. $\sup _{i}\left|\alpha_{i}(t, h)\right|=o(h)$.

Предполагаем также, что $q_{i, i+k}(t)=\lambda_{k}(t), q_{i, i-k}(t)=\mu_{k}(t)$ при $k>0$.

Другими словами, мы будем исследовать системы обслуживания, в которых интенсивности поступления и обслуживания $k$ требований в момент $t$ в системе обслуживания $\left(\lambda_{k}(t)\right.$ и $\mu_{k}(t)$ соответственно) не зависят от числа требований, находящихся в системе в момент $t$, причем $\lambda_{k+1}(t) \leqslant \lambda_{k}(t)$ и $\mu_{k+1}(t) \leqslant \mu_{k}(t)$ при всех $k$ и почти при всех $t \geqslant 0$. Далее, в соответствии со стандартным подходом (см. подробное описание в работах [7], [15]-[17]) предположим дополнительно, что все интенсивности являются линейными комбинациями конечного числа локально интегрируемых на $[0, \infty)$ функций. Кроме того, будем предполагать, что почти при всех $t \geqslant 0$

$$
L_{\lambda}(t)+L_{\mu}(t)=L(t)<\infty, \quad \text { где } \quad L_{\lambda}(t)=\sum_{i=1}^{\infty} \lambda_{i}(t), \quad L_{\mu}(t)=\sum_{i=1}^{\infty} \mu_{i}(t) .
$$

Тогда для описания вероятностной динамики процесса получаем прямую систему Колмогорова в виде дифференциального уравнения в пространстве последовательностей $l_{1}$ :

$$
\frac{d \mathbf{p}}{d t}=A(t) \mathbf{p}(t)
$$


где

$$
A(t)=\left(\begin{array}{cccccccc}
a_{00}(t) & \mu_{1}(t) & \mu_{2}(t) & \mu_{3}(t) & \mu_{4}(t) & \ldots & \mu_{r}(t) & \ldots \\
\lambda_{1}(t) & a_{11}(t) & \mu_{1}(t) & \mu_{2}(t) & \mu_{3}(t) & \ldots & \mu_{r-1}(t) & \ldots \\
\lambda_{2}(t) & \lambda_{1}(t) & a_{22}(t) & \mu_{1}(t) & \mu_{2}(t) & \ldots & \mu_{r-2}(t) & \ldots \\
\ldots & \ldots & \ldots & \ldots & \ldots & \ldots & \ldots & \ldots \\
\lambda_{r}(t) & \lambda_{r-1}(t) & \lambda_{r-2}(t) & \ldots & \lambda_{2}(t) & \lambda_{1}(t) & a_{r r}(t) & \ldots \\
\ldots & \ldots & \ldots & \ldots & \ldots & \ldots & \ldots & \ldots
\end{array}\right)
$$

причем $a_{i i}(t)=-\sum_{k=1}^{i} \mu_{k}(t)-\sum_{k=1}^{\infty} \lambda_{k}(t)$.

Далее $\|\cdot\|$ будет обозначать $l_{1}$-норму, т.е. $\|\mathbf{x}\|=\sum\left|x_{i}\right|$, a $\|B\|=\sup _{j} \sum_{i}\left|b_{i j}\right|$, если $B=\left(b_{i j}\right)_{i, j=0}^{\infty}$, и пусть $\Omega-$ множество всех векторов из $l_{1}$ с неотрицательными координатами и единичной нормой.

Тогда $\|A(t)\| \leqslant 2 \sum_{k=1}^{\infty}\left(\lambda_{k}(t)+\mu_{k}(t)\right) \leqslant 2 L(t)$ почти при всех $t \geqslant 0$, следовательно, операторная функция $A(t)$ ограничена почти при всех $t \geqslant 0$ и локально интегрируема на $[0 ; \infty)$.

Как известно (см., например, [8]), задача Коши для уравнения (1) тогда имеет единственное решение при любых начальных условиях, и из $\mathbf{p}(s) \in \Omega$ вытекает $\mathbf{p}(t) \in$ $\Omega$ при $t \geqslant s \geqslant 0$.

Далее $E(t, k)=\mathbf{E}\{X(t) \mid X(0)=k\}$ будет обозначать математическое ожидание процесса (среднее число требований) в момент $t$ при условии, что в нулевой момент времени он находится в состоянии $k$.

Первые содержательные оценки для такой модели в случае конечного фазового пространства получены в [20]. В настоящей статье для более общей ситуации счетного пространства состояний будут приведены оценки скорости сходимости в случае слабой эргодичности (п. 2) и оценки, связанные с усечениями фазового пространства модели (п. 3).

2. Оценки скорости сходимости. Напомним вначале основные понятия, изучаемые далее.

Марковскую цепь $X(t)$ назовем слабо эргодичной, если при любых начальных условиях $\mathbf{p}^{*}(0) \in \Omega, \mathbf{p}^{* *}(0) \in \Omega$ справедливо предельное соотношение $\lim _{t \rightarrow \infty} \| \mathbf{p}^{*}(t)-$ $\mathbf{p}^{* *}(t) \|=0$. В этом случае любое $\mathbf{p}^{*}(t)$ называется квазистачионарньл распределением марковской цепи $X(t)$.

Марковскую цепь назовем $X(t)$ эргодичной (сильно эргодичной), если существует вектор $\boldsymbol{\pi} \in \Omega$ такой, что $\lim _{t \rightarrow \infty}\|\mathbf{p}(t)-\boldsymbol{\pi}\|=0$ при любом $\mathbf{p}(0)=\mathbf{p} \in \Omega$. При этом вектор $\boldsymbol{\pi}$ называется стационарным распределением марковской цепи $X(t)$.

Марковская цепь $X(t)$ имеет предельное среднее $\phi(t)$, если при любом $k$ выполнено условие $|E(t, k)-\phi(t)| \rightarrow 0$ при $t \rightarrow \infty$.

Детальное обсуждение этих понятий проведено в [17].

Рассмотрим вспомогательную последовательность положительных чисел $\left\{d_{i}\right\}, i=$ $1,2, \ldots$, и треугольную матрицу $D$ следующего вида:

$$
D=\left(\begin{array}{cccc}
d_{1} & d_{1} & d_{1} & \cdots \\
0 & d_{2} & d_{2} & \cdots \\
0 & 0 & d_{3} & \cdots \\
\vdots & \ddots & \ddots & \ddots
\end{array}\right)
$$

Далее помимо исходного пространства $l_{1}$ будут использоваться еще два пространства последовательностей: $l_{1 E}=\left\{z=\left(p_{1}, p_{2}, \ldots\right)^{T}\left|\|z\|_{1 E} \equiv \sum n\right| p_{n} \mid<\infty\right\}$ и «основное» пространство $l_{1 D}=\left\{\mathbf{z}=\left(p_{1}, p_{2}, \ldots\right)^{T} \mid\|\mathbf{z}\|_{1 D} \equiv\|D \mathbf{z}\|_{1}<\infty\right\}$.

Положим

$$
d=\inf _{i \geqslant 1} d_{i}, \quad W=\inf _{k} \frac{d_{k}}{k} .
$$


Рассмотрим выражения

$$
\begin{gathered}
\alpha_{i}(t)=-a_{i i}(t)-\sum_{k=1}^{i-1}\left(\mu_{i-k}(t)-\mu_{i}(t)\right) \frac{d_{k}}{d_{i}}-\sum_{k \geqslant 1} \lambda_{k}(t) \frac{d_{k+i}}{d_{i}}, \\
\alpha(t)=\inf _{i \geqslant 1} \alpha_{i}(t), \quad \beta(t)=\sup _{i \geqslant 1} \alpha_{i}(t) .
\end{gathered}
$$

Положим

$$
\zeta_{i}(t)=-a_{i i}(t)+\sum_{k=1}^{i-1}\left(\mu_{i-k}(t)-\mu_{i}(t)\right) \frac{d_{k}}{d_{i}}+\sum_{k \geqslant 1} \lambda_{k}(t) \frac{d_{k+i}}{d_{i}}
$$

а также

$$
\xi(t)=\sup _{i \geqslant 1} \zeta_{i}(t)
$$

Теорема 1. Пусть существует последовательность положительных чисел $\left\{d_{j}\right\}$ maкas, umo $d>0 u$

$$
\int_{0}^{\infty} \alpha(t) d t=+\infty
$$

Тогда $X(t)$ слабо эргодичен и при любых начальньх условиях $\mathbf{p}^{*}(s), \mathbf{p}^{* *}(s)$ и любых $s, t, 0 \leqslant s \leqslant t$, справедлива оченка

$$
e^{-\int_{s}^{t} \xi(u) d u}\left\|\mathbf{p}^{*}(s)-\mathbf{p}^{* *}(s)\right\|_{1 D} \leqslant\left\|\mathbf{p}^{*}(t)-\mathbf{p}^{* *}(t)\right\|_{1 D} \leqslant e^{-\int_{s}^{t} \alpha(u) d u}\left\|\mathbf{p}^{*}(s)-\mathbf{p}^{* *}(s)\right\|_{1 D}
$$

Кроме того, если начальнье условия таковы, ито $D\left(\mathbf{p}^{*}(s)-\mathbf{p}^{* *}(s)\right) \geqslant 0$, то при любых $s, t, 0 \leqslant s \leqslant t$, справедлива оченка

$$
\left\|\mathbf{p}^{*}(t)-\mathbf{p}^{* *}(t)\right\|_{1 D} \geqslant e^{-\int_{s}^{t} \beta(u) d u}\left\|\mathbf{p}^{*}(s)-\mathbf{p}^{* *}(s)\right\|_{1 D} .
$$

Д о к а з а т е л ь с т в о. Общий подход к исследованию в случае процессов рождения и гибели подробно описан в [15], [17], для конечномерного стационарного случая см. также обсуждение в [9]. Те же методы удается применить и в рассматриваемой в настоящей работе ситуации, поэтому детали рассуждений здесь опущены.

Прежде всего положим $p_{0}=1-\sum_{i \geqslant 1} p_{i}$. Тогда из уравнения (1) получим

$$
\frac{d \mathbf{z}}{d t}=B(t) \mathbf{z}(t)+\mathbf{f}(t)
$$

где $\mathbf{f}(t)=\left(\lambda_{1}, \lambda_{2}, \ldots\right)^{T}$ и

$$
B=\left(\begin{array}{cccccccc}
a_{11}-\lambda_{1} & \mu_{1}-\lambda_{1} & \mu_{2}-\lambda_{1} & \mu_{3}-\lambda_{1} & \ldots & \ldots & \mu_{r-1}-\lambda_{1} & \ldots \\
\lambda_{1}-\lambda_{2} & a_{22}-\lambda_{2} & \mu_{1}-\lambda_{2} & \mu_{2}-\lambda_{2} & \ldots & \ldots & \mu_{r-2}-\lambda_{2} & \ldots \\
\lambda_{2}-\lambda_{3} & \lambda_{1}-\lambda_{3} & a_{33}-\lambda_{3} & \mu_{1}-\lambda_{2} & \ldots & \ldots & \mu_{r-3}-\lambda_{3} & \ldots \\
\ldots & \ldots & \ldots & \ldots & \ldots & \ldots & \ldots & \ldots \\
\lambda_{r-1}-\lambda_{r} & \lambda_{r-2}-\lambda_{r} & \ldots & \ldots & \lambda_{2}-\lambda_{r} & \lambda_{1}-\lambda_{r} & a_{r r}-\lambda_{r} & \ldots \\
\ldots & \ldots & \ldots & \ldots & \ldots & \ldots & \ldots & \ldots
\end{array}\right)
$$

Рассмотрим (5) как дифференциальное уравнение в пространстве $l_{1 D}$, при этом $B(t)$ и $\mathbf{f}(t)$ локально интегрируемы при $t \geqslant 0$.

Оценим логарифмическую норму

$$
\gamma(B(t))_{1 D}=\gamma\left(D B(t) D^{-1}\right)_{1} .
$$


Имеем

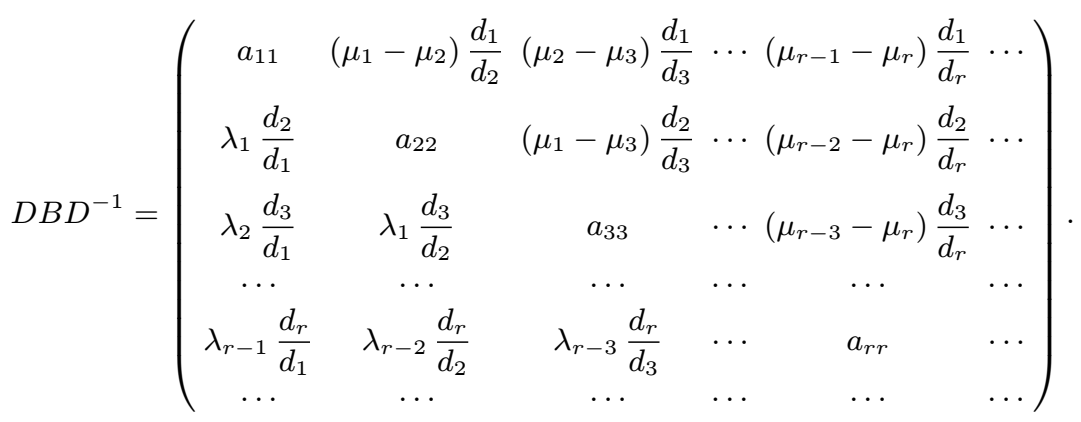

Получаем

$$
\begin{aligned}
\gamma(B(t))_{1 D} & =\sup \left(a_{i i}(t)+\sum_{k=1}^{i-1}\left(\mu_{i-k}(t)-\mu_{i}(t)\right) \frac{d_{k}}{d_{i}}+\sum_{k \geqslant 1} \lambda_{k}(t) \frac{d_{k+i}}{d_{i}}\right) \\
& =-\inf \alpha_{i}(t)=-\alpha(t) .
\end{aligned}
$$

Аналогично находим

$$
\gamma(-B(t))_{1 D}=\sup \zeta_{i}(t)=\xi(t) .
$$

Отсюда, как известно, и вытекает двухсторонняя оценка (3).

Для доказательства оценки (4) положим $\mathbf{v}(t)=D\left(\mathbf{p}^{*}(t)-\mathbf{p}^{* *}(t)\right)$. Тогда

$$
\frac{d \mathbf{v}}{d t}=D B(t) D^{-1} \mathbf{v}
$$

Все внедиагональные элементы матрицы $D B(t) D^{-1}$ неотрицательны, значит, из неотрицательности $\mathbf{v}(s)$ следует неотрицательность $\mathbf{v}(t)$ при всех $t \geqslant s$. А значит,

$$
\frac{d \sum v_{i}}{d t} \geqslant-\beta(t) \sum v_{i}
$$

откуда и вытекает неравенство (4).

Следствие 1. Пусть при выполнении условий теоремь 1 найдутся положительнье $M$, а такие, ито $e^{-\int_{s}^{t} \alpha(u) d u} \leqslant M e^{-a(t-s)}$ для всех $0 \leqslant s \leqslant t$. Тогда $X(t)$ экспоненциально эргодичен в норме пространства $l_{1 D}$, т.е. при любых начальных условиях $\mathbf{p}^{*}(s), \mathbf{p}^{* *}(s)$ и любых $s, t, 0 \leqslant s \leqslant t$, справедлива следуюшая оченка скорости сходимости:

$$
\left\|\mathbf{p}^{*}(t)-\mathbf{p}^{* *}(t)\right\|_{1 D} \leqslant M e^{-a(t-s)}\left\|\mathbf{p}^{*}(s)-\mathbf{p}^{* *}(s)\right\|_{1 D} .
$$

Следствие 2. При выполнении условий теоремь 1 справедлива следующая оценка скорости сходимости:

$$
\left\|\mathbf{p}^{*}(t)-\mathbf{p}^{* *}(t)\right\|_{1} \leqslant \frac{4}{d} e^{-\int_{s}^{t} \alpha(u) d u} \sum_{i \geqslant 1} g_{i}\left|p_{i}^{*}(s)-p_{i}^{* *}(s)\right|
$$

при любых начальных условиях $\mathbf{p}^{*}(s), \mathbf{p}^{* *}(s)$ и любых $s, t, 0 \leqslant s \leqslant t$, где $g_{i}=\sum_{n=1}^{i} d_{n}$.

Д о к а з а т е л ь с т в о. Достаточно воспользоваться оценками из [17], позволяющими сравнить рассматриваемые нормы:

$$
\begin{aligned}
\|\mathbf{z}\|_{1 D} & =d_{1}\left|\sum_{i=1}^{\infty} p_{i}\right|+d_{2}\left|\sum_{i=2}^{\infty} p_{i}\right|+d_{3}\left|\sum_{i=3}^{\infty} p_{i}\right|+\cdots \\
& \geqslant d\left(\left|\sum_{i=1}^{\infty} p_{i}\right|+\left|\sum_{i=2}^{\infty} p_{i}\right|+\left|\sum_{i=3}^{\infty} p_{i}\right|+\cdots\right)
\end{aligned}
$$




$$
\begin{aligned}
& \geqslant \frac{d}{2}\left(\left(\left|\sum_{i=1}^{\infty} p_{i}\right|+\left|\sum_{i=2}^{\infty} p_{i}\right|\right)+\left(\left|\sum_{i=2}^{\infty} p_{i}\right|+\left|\sum_{i=3}^{\infty} p_{i}\right|\right)+\cdots\right) \\
& \geqslant \frac{d}{2} \sum_{i=1}^{\infty}\left|p_{i}\right|=\frac{d}{2}\|\mathbf{z}\|_{1},
\end{aligned}
$$

и, значит,

$$
\begin{aligned}
\left\|\mathbf{p}^{*}(t)-\mathbf{p}^{* *}(t)\right\|_{1} & \leqslant 2\left\|\mathbf{z}^{*}(t)-\mathbf{z}^{* *}(t)\right\|_{1} \leqslant \frac{4}{d}\left\|\mathbf{z}^{*}(t)-\mathbf{z}^{* *}(t)\right\|_{1 D} \\
& \leqslant \frac{4}{d} e^{-\int_{s}^{t} \alpha(u) d u}\left\|\mathbf{z}^{*}(s)-\mathbf{z}^{* *}(s)\right\|_{1 D} \\
& \leqslant \frac{4}{d} e^{-\int_{s}^{t} \alpha(u) d u} \sum_{i \geqslant 1} g_{i}\left|p_{i}^{*}(s)-p_{i}^{* *}(s)\right| .
\end{aligned}
$$

Теорема 2. Пусть при выполнении условий предыдущей теоремы последовательность положительньх иисел $\left\{d_{j}\right\}$ такова, что $W>0$. Тогда $X(t)$ имеет предельное среднее $\phi(t)$ и при любых $k$ u $t \geqslant 0$ справедливо неравенство

$$
|E(t, k)-\phi(t)| \leqslant \frac{2 g_{k}}{W} e^{-\int_{0}^{t} \alpha(u) d u} .
$$

Д о к а з а т е л ь с т в о. Используя оценки из [17] для сравнения соответствующих норм, получаем

$$
\|\mathbf{z}\|_{1 D}=d_{1}\left|\sum_{i=1}^{\infty} p_{i}\right|+d_{2}\left|\sum_{i=2}^{\infty} p_{i}\right|+\cdots \geqslant W \sum_{k \geqslant 1} k\left|\sum_{i \geqslant k} p_{i}\right| \geqslant \frac{W}{2}\|\mathbf{z}\|_{1 E}
$$

вместо (7). Теперь, выбирая в качестве предельного среднего $\phi(t)=E(t, 0)$ и соответствующие $\mathbf{p}^{*}(t), \mathbf{p}^{* *}(t)$, получаем при произвольном $k \geqslant 0$ :

$$
\begin{aligned}
|E(t, k)-\phi| & \leqslant\left\|\mathbf{p}^{*}(t)-\mathbf{p}^{* *}(t)\right\|_{1 E} \\
& \leqslant \frac{2}{W}\left\|\mathbf{p}^{*}(t)-\mathbf{p}^{* *}(t)\right\|_{1 D} \leqslant \frac{2}{W} e^{-\int_{0}^{t} \alpha(u) d u}\left\|\mathbf{e}_{k}-\mathbf{e}_{0}\right\|_{1 D}=\frac{2 g_{k}}{W} e^{-\int_{0}^{t} \alpha(u) d u} .
\end{aligned}
$$

Рассмотрим теперь важнейшие частные случаи.

Теорема 3. Пусть $X(t)$ стационарна (т.е. интенсивности $\lambda_{k}$ и $\mu_{k}$ не зависят oт $t$ ) и существует последовательность положительных чисел $\left\{d_{j}\right\}$ такая, что $d>0$ и $\alpha=\inf _{i \geqslant 1} \alpha_{i}>0$. Тогда $X(t)$ сильно эргодичен (со стачионарным распределением $\boldsymbol{\pi})$ и при любом начальном условии $\mathbf{p}^{*}(0)$ и любом $t \geqslant 0$ справедливь оченки

$$
e^{-\xi t}\left\|\mathbf{p}^{*}(0)-\boldsymbol{\pi}\right\|_{1 D} \leqslant\left\|\mathbf{p}^{*}(t)-\boldsymbol{\pi}\right\|_{1 D} \leqslant e^{-\alpha t}\left\|\mathbf{p}^{*}(0)-\boldsymbol{\pi}\right\|_{1 D}
$$

$$
\left\|\mathbf{p}^{*}(t)-\boldsymbol{\pi}\right\|_{1} \leqslant \frac{4}{d} e^{-\alpha t} \sum_{i \geqslant 1} g_{i}\left|p_{i}^{*}(0)-\pi_{i}\right| .
$$

Кроме того, если начальное условие таково, что $D\left(\mathbf{p}^{*}(0)-\boldsymbol{\pi}\right) \geqslant 0$, то при любом $t \geqslant 0$ справедлива оченка

$$
\left\|\mathbf{p}^{*}(t)-\boldsymbol{\pi}\right\|_{1 D} \geqslant e^{-\beta t}\left\|\mathbf{p}^{*}(0)-\boldsymbol{\pi}\right\|_{1 D} .
$$

3 а м е ч а н и е 1. Аналогично теореме 2, если выполнено дополнительное условие $W>0$, получаются результат о предельном среднем и оценка скорости сходимости к нему. Однако при этом теперь в качестве предельного среднего естественно выбрать постоянное $\phi=\sum k \pi_{k}$, соответствующее стационарному распределению. При этом оценка скорости сходимости выглядит следующим образом:

$$
|E(t, k)-\phi| \leqslant \frac{2}{W} e^{-\alpha t}\left\|\mathbf{p}^{*}(0)-\boldsymbol{\pi}\right\|_{1 D} .
$$


Теорема 4. Если интенсивности поступления и обслуживания требований $\lambda_{k}$ и $\mu_{k}$ являются 1-периодическими по $t$ и существует последовательность положительньх чисел $\left\{d_{j}\right\}$ такая, что $d>0$, а $\alpha^{*}=\int_{0}^{1} \alpha(t) d t>0$, то соответствуюшие предельный режим $\mathbf{p}^{* *}(t)$ и предельное среднее $\phi(t)$ (если $W>0$ ) можно выбрать также 1-периодическими. При этом прочесс будет вдобавок экспоненциально эргодичнылм и можно положить $M=\exp \left(\sup _{|t-s| \leqslant 1} \int_{s}^{t} \alpha(u) d u\right), a=\alpha^{*}$.

Рассмотрим теперь отдельно уточнения, появляющиеся в том случае, когда пространство состояний конечно $\left(E_{N}=\{0,1, \ldots, N\}\right)$.

Отметим прежде всего, что вид матрицы (6) при этом, вообще говоря, изменится, а именно, будет следующим:

$$
\begin{aligned}
& D B D^{-1} \\
& =\left(\begin{array}{ccccc}
a_{11}-\lambda_{N} & \left(\mu_{1}-\mu_{2}\right) \frac{d_{1}}{d_{2}} & \left(\mu_{2}-\mu_{3}\right) \frac{d_{1}}{d_{3}} & \cdots & \left(\mu_{N-1}-\mu_{N}\right) \frac{d_{1}}{d_{N}} \\
\left(\lambda_{1}-\lambda_{N}\right) \frac{d_{2}}{d_{1}} & a_{22}-\lambda_{N-1} & \left(\mu_{1}-\mu_{3}\right) \frac{d_{2}}{d_{3}} & \cdots & \left(\mu_{N-2}-\mu_{N}\right) \frac{d_{2}}{d_{N}} \\
\left(\lambda_{2}-\lambda_{N}\right) \frac{d_{3}}{d_{1}} & \left(\lambda_{1}-\lambda_{N-1}\right) \frac{d_{3}}{d_{2}} & a_{33}-\lambda_{N-2} & \cdots & \left(\mu_{N-3}-\mu_{N}\right) \frac{d_{3}}{d_{N}} \\
\ldots & \ldots & \ldots & \ldots & \cdots \\
\left(\lambda_{N-1}-\lambda_{N}\right) \frac{d_{N}}{d_{1}} & \left(\lambda_{N-2}-\lambda_{N-1}\right) \frac{d_{N}}{d_{2}} & \left(\lambda_{N-3}-\lambda_{N-2}\right) \frac{d_{N}}{d_{3}} & \ldots & a_{N N}-\lambda_{1}
\end{array}\right) .
\end{aligned}
$$

Соответственно изменятся и выражения для $\alpha_{i}(t)$ и $\zeta_{i}(t)$.

При этом $\|\mathbf{z}\|_{1 D}=d_{1}\left|\sum_{i=1}^{N} p_{i}\right|+d_{2}\left|\sum_{i=2}^{N} p_{i}\right|+\cdots+d_{N}\left|p_{N}\right| \leqslant g_{N}\|\mathbf{z}\|_{1}$, так что вместо теоремы 1 получаем следующее утверждение.

Теорема 5. Пусть для системь с конечныл фазовым пространством $E_{N}$ существует последовательность положительных чисел $\left\{d_{j}\right\}$ такая, что выполнено условие (2). Тогда $X(t)$ слабо эргодичен и при любьх начальньх условиях $\mathbf{p}^{*}(s)$, $\mathbf{p}^{* *}(s)$ и любых $s, t, 0 \leqslant s \leqslant t$, справедлива оченка

$$
\begin{aligned}
& \frac{d}{2 g_{N}} e^{-\int_{s}^{t} \xi(u) d u}\left\|\mathbf{p}^{*}(s)-\mathbf{p}^{* *}(s)\right\|_{1} \\
& \quad \leqslant\left\|\mathbf{p}^{*}(t)-\mathbf{p}^{* *}(t)\right\|_{1} \leqslant \frac{4 g_{N}}{d} e^{-\int_{s}^{t} \alpha(u) d u}\left\|\mathbf{p}^{*}(s)-\mathbf{p}^{* *}(s)\right\|_{1} .
\end{aligned}
$$

Кроме того, если начальные условия таковы, что $D\left(\mathbf{p}^{*}(s)-\mathbf{p}^{* *}(s)\right) \geqslant 0$, то при любих $s, t, 0 \leqslant s \leqslant t$, справедлива оченка

$$
\left\|\mathbf{p}^{*}(t)-\mathbf{p}^{* *}(t)\right\|_{1} \geqslant \frac{d}{2 g_{N}} e^{-\int_{s}^{t} \beta(u) d u}\left\|\mathbf{p}^{*}(s)-\mathbf{p}^{* *}(s)\right\|_{1} .
$$

3 а м е ч а н и е 2. Отметим, что если удается выбрать $d_{i}$ так, чтобы функции $\alpha(t)$ и $\beta(t)$ оказались одинаковыми (т.е. $\alpha_{i}(t)$ не зависят от $i$ ), то получится точная по порядку оценка скорости сходимости.

3 а м е ч а н и е 3. Если вдобавок интенсивности поступления и обслуживания требований постоянны, причем $\lambda_{1}>\lambda_{2}$ и $\mu_{1}>\mu_{2}$, то, как вытекает из теоремы 9 в [17] (см. также [9, следствие 1]), существует последовательность $\left\{d_{i}\right\}$, удовлетворяющая условиям предыдущего замечания (т.е. такая, что $\alpha=\beta$ ). Тем самым, эта последовательность дает точное значение параметра сходимости.

3. Аппроксимация. Рассмотрим здесь задачу о построении предельного среднего с помощью аппроксимации системой с конечным (или меньшим) фазовым пространством. Впервые такая методика для простейших ситуаций была описана в [13], а построение различных предельных характеристик для нестационарных процессов рождения и гибели с помощью аппроксимаций конечными процессами рассматривалось в [16], [19]. 
Для простоты будем предполагать в этом пункте, что почти при всех $t \geqslant 0$ выполнены следующие условия:

$$
\begin{aligned}
& L_{\lambda}(t) \leqslant L_{\lambda}<\infty, \quad L_{\mu}(t) \leqslant L_{\mu}<\infty, \quad \lambda_{k}(t)=0 \quad \text { при } k>r, \\
& \|\mathbf{f}(t)\|_{1 D}=d_{1} \lambda_{1}(t)+\left(d_{1}+d_{2}\right) \lambda_{2}(t)+\cdots=\sum_{i \geqslant 1} g_{i} \lambda_{i}(t) \leqslant K<\infty .
\end{aligned}
$$

Теперь в предположении экспоненциальной эргодичности процесса можно получить оценку

$\|\mathbf{z}(t)\|_{1 D} \leqslant\|V(t)\|_{1 D}\|\mathbf{z}(0)\|_{1 D}+\int_{0}^{t}\|V(t, \tau)\|_{1 D}\|\mathbf{z}(\tau)\|_{1 D} d \tau \leqslant M e^{-a t}\|\mathbf{z}(0)\|_{1 D}+\frac{K M}{a}$,

где $V(t, \tau)$ - оператор Коши уравнения (5).

Значит, если взять $X(0)=0$, то соответственно $\mathbf{p}(0)=(1,0,0, \ldots)^{T}, \mathbf{z}(0)=\mathbf{0}$, а тогда будет выполнено неравенство

$$
\|\mathbf{z}(t)\|_{1 D} \leqslant \frac{K M}{a} .
$$

Рассматривая теперь соответствующее решение уравнения (1) и предполагая, соответственно, что все $p_{i}(t)$ неотрицательны, получаем

$$
\|z(t)\|_{1 D}=\sum_{i \geqslant 1} p_{i}(t) \sum_{k=1}^{i} d_{k} \geqslant\left(d_{1}+\cdots+d_{n}\right) \sum_{i \geqslant n} p_{i}(t)=g_{n} \sum_{i \geqslant n} p_{i}(t),
$$

откуда

$$
\sum_{i=n}^{\infty} p_{i}(t) \leqslant \frac{K M}{a g_{n}} .
$$

3 а м е ч а н и е 4. Отметим, что из (9) вытекает следующая оценка числа требований в системе:

$$
\operatorname{Pr}\{X(t)<n\} \geqslant 1-\frac{K M}{a g_{n}} .
$$

Первые оценки такого рода были получены в [14].

Введем следующие выражения:

$$
\omega(m, n)=\sup _{k \geqslant 0} \frac{m+k}{g_{n+k}}, \quad m, n \geqslant 0 .
$$

Рассмотрим теперь «усеченный» процесс $X_{N-1}(t)$ с состояниями от 0 до $N-1$, заданный соответствующей матрицей

$$
A_{N-1}(t)=\left(\begin{array}{ccccccc}
b_{00} & \mu_{1} & \mu_{2} & \mu_{3} & \mu_{4} & \ldots & \mu_{N-1} \\
\lambda_{1} & b_{11} & \mu_{1} & \mu_{2} & \mu_{3} & \ldots & \mu_{N-2} \\
\lambda_{2} & \lambda_{1} & b_{22} & \mu_{1} & \mu_{2} & \ldots & \mu_{N-3} \\
\ldots & \ldots & \ldots & \ldots & \ldots & \ldots & \ldots \\
\lambda_{N-1} & \lambda_{N-2} & \lambda_{N-3} & \ldots & \lambda_{2} & \lambda_{1} & b_{N-1, N-1}
\end{array}\right),
$$

причем $b_{i i}(t)=-\sum_{k=1}^{i} \mu_{k}(t)-\sum_{k=1}^{N-1-i} \lambda_{k}(t)$.

Векторы вероятностей состояний для исходного и усеченного процесса описываются соответственно уравнениями (1) и

$$
\frac{d \mathbf{p}^{*}}{d t}=A_{N-1}(t) \mathbf{p}^{*}
$$

Перепишем (1) в следующей форме (отождествляя конечномерный вектор и соответствующий ему счетный вектор с теми же «начальными» ненулевыми координатами):

$$
\frac{d \mathbf{p}}{d t}=A_{N-1}(t) \mathbf{p}+\left(A(t)-A_{N-1}(t)\right) \mathbf{p}
$$


Тогда получаем

$$
\mathbf{p}(t)=U_{N-1}(t) \mathbf{p}(0)+\int_{0}^{t} U_{N-1}(t, \tau)\left(A(\tau)-A_{N-1}(\tau)\right) \mathbf{p}(\tau) d \tau
$$

$$
\mathbf{p}^{*}(t)=U_{N-1}(t) \mathbf{p}^{*}(0)
$$

где $U_{N-1}(t, \tau)$ - оператор Коши для уравнения (10).

Будем считать, что $\mathbf{p}(0)=\mathbf{p}^{*}(0)$. Тогда

$$
\mathbf{p}(t)-\mathbf{p}^{*}(t)=\int_{0}^{t} U_{N-1}(t, \tau)\left(A(\tau)-A_{N-1}(\tau)\right) \mathbf{p}(\tau) d \tau
$$

Оценим $\left\|U_{N-1}(t, \tau)\left(A(\tau)-A_{N-1}(\tau)\right) \mathbf{p}(\tau)\right\|_{1 E}$.

Запишем

$$
\begin{gathered}
A(t)-A_{N-1}(t) \\
\quad\left(\begin{array}{ccllclll}
C_{0,0}(t) & 0 & \ldots & 0 & 0 & \ldots & \mu_{N}(t) & \ldots \\
0 & C_{1,1}(t) & \ldots & 0 & 0 & \ldots & \mu_{N-1}(t) & \ldots \\
0 & 0 & \ldots & 0 & 0 & \ldots & \mu_{N-2}(t) & \ldots \\
\ldots & \ldots & \ldots & \ldots & \ldots & \ldots & \ldots & \ldots \\
0 & 0 & \ldots & C_{N-r, N-r}(t) & 0 & \ldots & \mu_{r}(t) & \ldots \\
0 & 0 & \ldots & 0 & C_{N-r+1, N-r+1}(t) & \ldots & \mu_{r-1}(t) & \ldots \\
0 & 0 & \ldots & 0 & 0 & \ldots & \mu_{r-2}(t) & \ldots \\
\ldots & \ldots & \ldots & \ldots & \ldots & \ldots & \ldots & \\
\lambda_{N}(t) & \lambda_{N-1}(t) & \ldots & \lambda_{r}(t) & \lambda_{r-1}(t) & \ldots & a_{N, N} & \ldots \\
\ldots & \ldots & \ldots & \ldots & \ldots & \ldots & \ldots & \ldots
\end{array}\right) .
\end{gathered}
$$

Тогда

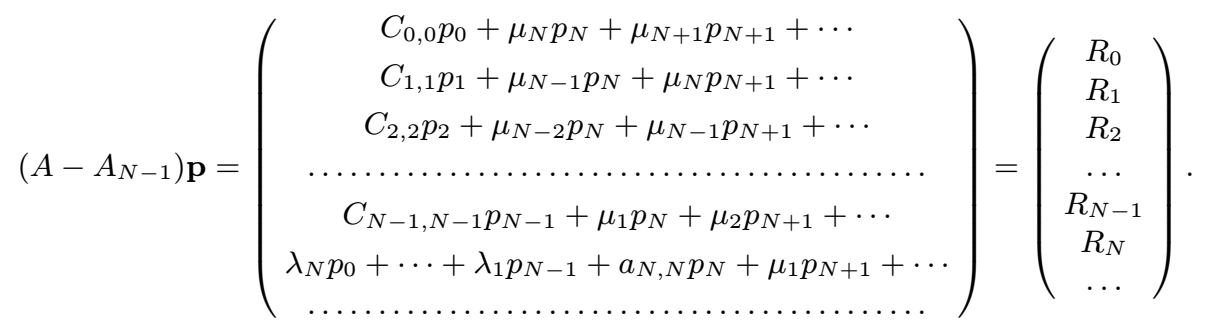

Далее, отметим, что

\section{А тогда}

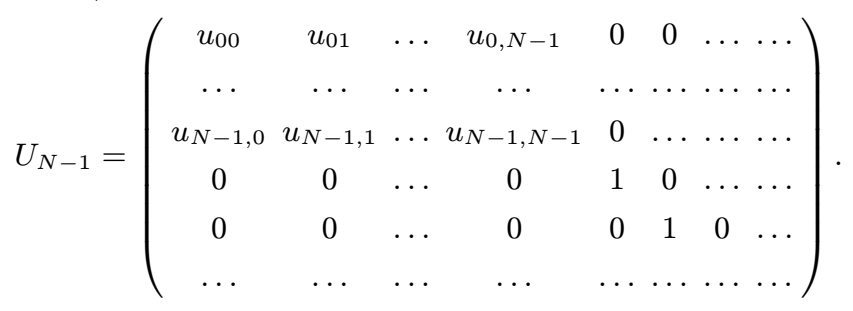

$$
\begin{aligned}
& \left\|U_{N-1}\left(A-A_{N-1}\right) \mathbf{p}\right\|_{1 E} \\
& \quad \leqslant(N-1)\left(\left|R_{1}\right|+\left|R_{2}\right|+\cdots+\left|R_{N-1}\right|\right)+N\left|R_{N}\right|+(N+1)\left|R_{N+1}\right|+\cdots \\
& \quad \leqslant(N-1)\left(\left|R_{1}\right|+\left|R_{2}\right|+\cdots+\left|R_{N-1}\right|\right)+N\left|a_{N, N}\right| p_{N}+(N+1)\left|a_{N+1, N+1}\right| p_{N+1}+\cdots \\
& \quad \leqslant \mu_{1}\left(N p_{N+1}+(N+1) p_{N+2}+\cdots\right)+\mu_{2}\left(N p_{N+2}+(N+1) p_{N+3}+\cdots\right)+\cdots \\
& \quad \leqslant \lambda_{1}\left(N p_{N-1}+(N+1) p_{N}+\cdots\right)+\cdots+\lambda_{r}\left(N p_{N-r}+(N+1) p_{N-r+1}+\cdots\right) .
\end{aligned}
$$


Рассмотрим отдельно каждую группу слагаемых в правой части последнего неравенства, предполагая, что $N>r$. Тогда $\left|C_{k k}(t)\right|=0$ при $k<N-r$.

Первая группа оценивается следующим образом:

$$
\begin{aligned}
& (N-1)\left(\left|R_{1}\right|+\left|R_{2}\right|+\cdots+\mid R_{N-1}\right) \mid \\
& \quad \leqslant(N-1)\left(\sum_{k=N-r+1}^{N-1}\left|C_{k k}\right| p_{k}+p_{N} \sum_{k=1}^{N} \mu_{k}+p_{N+1} \sum_{k=2}^{N+1} \mu_{k}+\cdots\right) \\
& \leqslant(N-1)\left(\lambda_{r} p_{N-r}+\left(\lambda_{r-1}+\lambda_{r}\right) p_{N-r+1}+\cdots+\left(\lambda_{r}+\cdots+\lambda_{1}\right) p_{N-1}+\frac{L_{\mu} K M}{a g_{N}}\right) \\
& \leqslant(N-1)\left(\frac{L_{\lambda} K M}{a g_{N-r}}+\frac{L_{\mu} K M}{a g_{N}}\right) .
\end{aligned}
$$

Чтобы оценить остальные выражения, отметим, что

$$
\sum_{k \geqslant 0}(m+k) p_{n+k} \leqslant \sum_{k \geqslant 0} \frac{m+k}{g_{n+k}} g_{n+k} p_{n+k} \leqslant \omega(m, n)\|\mathbf{z}\|_{1 D} \leqslant \frac{\omega(m, n) K M}{a} .
$$

Тогда

$$
N\left|a_{N, N}\right| p_{N}+(N+1)\left|a_{N+1, N+1}\right| p_{N+1}+\cdots \leqslant \frac{\omega(N, N) K M\left(L_{\lambda}+L_{\mu}\right)}{a} .
$$

Третья группа слагаемых оценивается следующим образом:

$$
\begin{aligned}
\mu_{1}\left(N p_{N+1}+\right. & \left.(N+1) p_{N+2}+\cdots\right)+\mu_{2}\left(N p_{N+2}+(N+1) p_{N+3}+\cdots\right)+\cdots \\
& \leqslant \frac{K M}{a} \sum_{k \geqslant 1} \mu_{k} \omega(N, N+k) .
\end{aligned}
$$

Аналогично,

$$
\begin{aligned}
\lambda_{1}\left(N p_{N-1}+\right. & \left.(N+1) p_{N}+\cdots\right)+\lambda_{2}\left(N p_{N-2}+(N+1) p_{N-1}+\cdots\right) \\
& +\cdots+\lambda_{r}\left(N p_{N-r}+(N+1) p_{N-r+1}+\cdots\right) \\
\leqslant & \frac{K M}{a} \sum_{k=1}^{r} \lambda_{k} \omega(N, N-k) .
\end{aligned}
$$

А тогда

$$
\begin{aligned}
\left|E(t, 0)-E_{N-1}(t, 0)\right| \leqslant \frac{K M t}{a}( & (N-1)\left(\frac{L_{\lambda}}{g_{N-r}}+\frac{L_{\mu}}{g_{N}}\right)+\omega(N, N)\left(L_{\lambda}+L_{\mu}\right) \\
& \left.+\sum_{k \geqslant 1} \mu_{k} \omega(N, N+k)+\sum_{k=1}^{r} \lambda_{k} \omega(N, N-k)\right)
\end{aligned}
$$

и получаем следующий результат.

Теорема 6. Пусть выполнены условия следствия 1 и теоремь 2 , а также условия (8). Тогда при всех $t \geqslant 0$ справедлива оченка (11).

3 а м е ч а н и е 5. Условия (8) можно, очевидно, существенно ослабить за счет усложнения оценок.

3 а м е ч а н и е 6 . В стандартных системах обслуживания вспомогательная последовательность $\left\{d_{i}\right\}$ является геометрической прогрессией со знаменателем больше единицы. При этом правая часть (11) быстро стремится к нулю при любом фиксированном $t$, если $N \rightarrow \infty$. 


\section{СПИСОК ЛИТЕРАТУРЫ}

1. Баруча-Рид А. Т. Элементы теории марковских процессов и их приложения. М.: Наука, 1969, $511 \mathrm{c.}$

2. Гнеденко Б. В., Макаров И.П. Свойства решений задачи с потерями в случае периодических интенсивностей. - Дифф. уравнения, 1971, т. 9, с. 1696-1698.

3. Гнеденко Б. В., Коваленко И. Н. Введение в теорию массового обслуживания. М.: Наука, 1987, $336 \mathrm{c.}$

4. Гнеденко Б. В., Соловьев А. Д. Об условиях существования финальных вероятностей марковского процесса. - Math. Operationsforsh. Statist., 1973, № 5, p. 379390.

5. Гнеденко Д. Б. Об одном обобщении формулы Эрланга. — Zastosow. Mat., 1971, v. 12 , p. $239-242$.

6. Granovsky B.L., Zeifman A.I. The N-limit of spectral gap of a class of birth-death Markov chains. - Appl. Stoch. Models Bus. Ind., 2000, v. 16, № 4, p. 235-248.

7. Granovsky B.L., Zeifman A.I. Nonstationary queues: estimation of the rate of convergence. - Queueing Syst., 2004, v. 46, № 3-4, p. 363-388.

8. Далецкий Ю. Л., Крейн М. Г. Устойчивость решений дифференциальных уравнений в банаховом пространстве. М.: Наука, 1970, 534 с.

9. Ван Доорн Э. А., Зейфман А. И., Панфилова Т. Л. Оценки и асимптотика скорости сходимости для процессов рождения и гибели. - Теория вероятн. и ее примен., 2009 , т. 54 , в. 1 , с. $18-38$.

10. van Doorn E.A., Zeifman A.I. On the speed of convergence to stationarity of the Erlang loss system. - Queueing Syst., 2009, v. 63, № 1-4, p. 241-252.

11. Mitrophanov A. Yu. Stability and exponential convergence of continuous-time Markov chains. - J. Appl. Probab., 2003, v. 40, № 4, p. 970-979.

12. Саати Т. Л. Элементы теории массового обслуживания и ее приложения. М.: Советское радио, 1971,520 с.

13. Зейфман А.И. О погрешности усечения системы рождения и гибели. - Журн. вычисл. матем. и матем. физ., 1988, т. 28, № 12, с. 1906-1907.

14. Zeifman A.I. On the estimation of probabilities for birth and death processes. - J. Appl. Probab., 1995, v. 32, № 3, p. 623-634.

15. Zeifman A.I. Upper and lower bounds on the rate of convergence for nonhomogeneous birth and death processes. — Stochastic Process. Appl., 1995, v. 59, № 1, p. 157-173.

16. Zeifman A., Leorato S., Orsingher E., Satin Ya., Shilova G. Some universal limits for nonhomogeneous birth and death processes. - Queueing Syst., 2006, v. 52, № 2, p. $139-151$.

17. Зейфман А.И., Бенинг В.Е., Соколов И. А. Марковские цепи и модели с непрерывным временем. М.: Элекс-КМ, 2008, 167 с.

18. Зейфман А.И. О нестационарной модели Эрланга. - Автомат. и телемех., 2009, № 12 , с. $71-80$.

19. Зейфман А.И., Коротылшева А. В., Панфилова Т. Л., Шоргин С. Я. Оценки устойчивости для некоторых систем обслуживания с катастрофами. - Информ. и ее примен., 2011, т. 5, № 3, с. 27-33.

20. Сатин Я.А., Зейфман А.И., Коротьишева А.В., Шоргин С.Я. Об одном классе марковских систем обслуживания. - Информ. и ее примен., 2011, т. 5, № 4, c. $6-12$.

21. Zeifman A., Korotysheva A. Perturbation bounds for $M_{t} / M_{t} / N$ queue with catastrophes. - Stoch. Models, 2012, v. 28, № 1, p. 49-62.

Поступила в редакцию 